International Mathematical Forum, 2, 2007, no. 51, 2519 - 2522

\title{
Base Locus of Linear Systems Containing a Fixed Finite Set
}

\author{
E. Ballico ${ }^{1}$ \\ Dept. of Mathematics \\ University of Trento \\ 38050 Povo (TN), Italy \\ ballico@science.unitn.it
}

\begin{abstract}
Let $S$ be a finite subset of a projective variety. Here we give some condition on a linear system $V$ on $X$ which imply that $S$ is the schemetheoretic base locus of $V$.
\end{abstract}

\section{Mathematics Subject Classification: 14N05}

Keywords: base locus

Let $S$ be a finite subset of a projective variety. Here we give some condition on a linear system $V$ on $X$ which imply that $S$ is the scheme-theoretic base locus of $V$. For a very refined study of this problem when $X=\mathbf{P}^{2}$, see [2]. We will first prove the following result.

Proposition 1. Fix integers $n, d, s$ such that $n \geq 2, d \geq 2, d^{n} \geq 25$, and $\left(\begin{array}{c}n+s-1 \\ \leq\end{array}\right) s \leq\left(\begin{array}{c}n+s \\ n\end{array}\right)-n-1$. Let $Z \subset \mathbf{P}^{n}$ a general complete intersection of $n$ degree d hypersurfaces. Let $S \subset Z$ be any subset such that $\sharp(S)=s$. Then $h^{0}\left(\mathbf{P}^{n}, \mathcal{I}_{S}(d-1)\right)=0, h^{1}\left(\mathbf{P}^{n}, \mathcal{I}_{S}(t)\right)=0$ for all $t \geq d, h^{0}\left(\mathbf{P}^{n}, \mathcal{I}_{S}(d)\right)=\left(\begin{array}{c}n+d \\ n\end{array}\right)-s$ and $S$ is the scheme-theoretic base locus of the linear system $\left|\mathcal{I}_{S}(d)\right|$.

The assumption $d^{n} \geq 25$ is satisfied, unless either $n=2$ and $d=2,3,4$ or $d=2$ and $n=3,4$.

An immediate consequence of Proposition 1 is the following result.

Corollary 1. Fix integers $n, d, s$ such that $n \geq 2, d \geq 2, d^{n} \geq 25$, and $\left(\begin{array}{c}n+s-1 \\ \leq\end{array}\right) s \leq\left(\begin{array}{c}n+s \\ n\end{array}\right)-n-1$. Let $S \subset \mathbf{P}^{n}$ be a general subset such that $\sharp(S)=s$. Then $h^{0}\left(\mathbf{P}^{n}, \mathcal{I}_{S}(d-1)\right)=0, h^{1}\left(\mathbf{P}^{n}, \mathcal{I}_{S}(t)\right)=0$ for all $t \geq d, h^{0}\left(\mathbf{P}^{n}, \mathcal{I}_{S}(d)\right)=$ $\left(\begin{array}{c}n+d \\ n\end{array}\right)-s$ and $S$ is the scheme-theoretic base locus of the linear system $\left|\mathcal{I}_{S}(d)\right|$.

\footnotetext{
${ }^{1}$ The author was partially supported by MIUR and GNSAGA of INdAM (Italy).
} 
Proposition 2. Fix integers $n, d, s$, e such that $n \geq 2, d \geq 2, e>0$, and $\left(\begin{array}{c}n+s-1 \\ -\end{array}\right) n \leq s \leq\left(\begin{array}{c}n+s \\ n\end{array}\right)-1$. Fix a general $\left(\begin{array}{c}n+s \\ n\end{array}\right)-s$ linear subspace $M$ of $H^{0}\left(\mathbf{P}^{n}, \mathcal{O}_{\mathbf{P}^{n}}(d)\right)$ and a general $\left(n-\left(\begin{array}{c}n+s \\ n\end{array}\right)+s\right)$-dimensional linear subspace of $H^{0}\left(\mathbf{P}^{n}, \mathcal{O}_{\mathbf{P}^{n}}(d+e)\right)$. Let $Z_{1}$ the scheme-theoretic intersection of the zero-loci of the homogeneous forms in $M$. Let $Z$ be the scheme-theoretic intersection of $Z$ and of the the zero-loci of the homogeneous forms in $M^{\prime} . Z_{1}$ is a smooth complete intersection of dimension $n-\left(\begin{array}{c}n+s \\ n\end{array}\right)+s$ and of degree $d^{\left(n-\left(\begin{array}{c}n+s \\ n\end{array}\right)+s\right)}$. Z is a finite set of $\gamma:=d^{\left(n-\left(\begin{array}{c}n+s \\ n\end{array}\right)+s\right)} \cdot(d+e)^{\left(\left(\begin{array}{c}n+s \\ n\end{array}\right)-s\right)}$ points. Assume $\gamma \geq 25$. Let $S \subset Z$ be any subset such that $\sharp(S)=s$. $S$ is the scheme-theoretic intersection of $Z_{1}$ and the base locus of the linear system $\left|\mathcal{I}_{S}(d+e)\right|$.

The assumption $\gamma \geq 25$ is satisfied if $d^{(n-1)} \cdot(d+e) \geq 25$. Now we fix $S$ and consider the stratification of $\mathbf{P}^{n} \backslash S$ by the base locus of $\mathcal{I}_{S \cup\{P\}}(d) \mid, P \in \mathbf{P}^{n} \backslash S$.

Theorem 1. Let $X$ be an integral $n$-dimensional projective variety and a $n$ very ample linear system (not necessarly complete) on $X$, say associated to the line bundle $L$. For any finite $A \subset X_{\text {reg }}$ let $\pi_{A}: X_{A} \rightarrow X$ denote the blowing up of $A$ and $E_{A}:=\cup_{P \in A} \pi_{A}^{-1}(P)$ (the union of all exceptional divisors). For every integer $x$ such that $1 \leq x \leq 6$ fix a general $A_{x} \subset X$ with $\sharp\left(A_{x}\right)=x$ and let $V_{A_{x}}, 1 \leq x \leq 6$, denote the linear system $\pi_{A_{x}}^{*}(V) \cap H^{0}\left(X_{A_{x}}, \pi_{A_{x}}^{*}(L)\left(-E_{A_{x}}\right)\right)$. Assume that all linear systems $V_{A_{x}}, 1 \leq x \leq 6$, are very ample. Fix an integer $s$ such that $1 \leq s \leq \operatorname{dim}(V)-n-1$, and a general $A \subset X$ such that $\sharp(A)=s$. Then the linear system $V_{A}$ on $X_{A}$ is base point free.

Remark 1. Let $C$ be an integral projective curve and $A$ a base point free linear system on $C$, say associated to the linear subspace $V \subseteq H^{0}(C, L)$, $L \in \operatorname{Pic}(C)$. For a general $P \in C$ the linear system $A(-P)$ associated to $V \cap H^{0}\left(C, \mathcal{I}_{P} \otimes L\right)$ is base point free if and only if the mprphism $h_{A}: C \rightarrow \mathbf{P}^{n}$, $n:=\operatorname{dim}(V)-1$, is birational onto its image. Assume that this is the case and fix an integer $a$ such that $1 \leq a \leq n-3$. Fix a general $\left(P_{1}, \ldots, P_{a}\right) \in\left(\mathbf{P}^{n}\right)^{a}$. In characteristic zero the linear projection of $h_{A}(C)$ from the linear subspace $\left\langle\left\{h_{A}\left(P_{1}\right), \ldots, h_{A}\left(P_{a}\right)\right\}\right\rangle$ is birational onto its image by the linear independence of a general hyperplane section $([1])$. Hence the linear system $A\left(-P_{1}-\cdots-\right.$ $\left.P_{a}\right)$ is base point free. In positive characteristic the existence of very strange projective curves shows that this is not always true.

Remark 2. Let $Z \subset \mathbf{P}^{n}$ a zero-dimensional scheme and $t$ a positive integer such that $h^{1}\left(\mathbf{P}^{n}, \mathcal{I}_{Z}(t)\right)=0$. By Castelnuovo-Mumford's lemma the homogeneous ideal of $Z$ is generated by forms of degree at most $t+1$. Hence $Z$ is the scheme-theoretic base locus of the linear system $\left|\mathcal{I}_{Z}(t+1)\right|$.

Proof of Proposition 1. Let $\Gamma_{n, d}$ denote the set of all $n$-dimensional linear subspaces $V \subset H^{0}\left(\mathbf{P}^{n}, \mathcal{O}_{\mathbf{P}^{n}}(d)\right)$ such that the scheme-theoretic base locus $B_{V}$ of $V$ is a disjoint union of $d^{n}$ points. Set $I:=\left\{(V, P) \in \Gamma_{n, d} \times \mathbf{P}^{n}\right.$ : $\left.P \in B_{V}\right\}$ (the incidence correspondence) and let $\pi: I \rightarrow \Gamma_{n, d}$ be the map 
induced by the projection on the first factor. $\pi$ is proper, étale and of degree $d^{n}$. Let $G$ be the Galois group of the finite map $\pi$. $G$ is well-defined, because $\Gamma_{n, d}$ is irreducible. Fixing any $W \in \Gamma_{n, d}$ and any ordering of the $d^{n}$ points in $B_{W}$ we may see $G$ as a subgroup of the symmetric group $S_{d^{n}}$. We assume that $W$ is general in $\Gamma_{n, d}$.

Claim: $G$ contains the alternating group $A_{d^{n}}$.

Proof of the Claim: By assumption $d^{n} \geq 25$. By [1], Th. 2.4, it is sufficient to show that $G$ is at least 5 -transitive. Let $\pi_{2}: I \rightarrow \mathbf{P}^{n}$ be the map induced by the second projection. $\pi_{2}$ is surjective and each fiber of $\pi_{2}$ is isomorphic to a vector space of dimension $\left(\begin{array}{c}n+d \\ n\end{array}\right)-1$. Hence $I$ is irreducible. The 1 -transitivity of $G$ is equivalent to the irreducibility of $I$. Fix an integer $x$ such that $2 \leq x \leq 6$ and assume that $G$ is $(s-1)$-transitive. We will show that $G$ is $x$ transitive. Fix $P_{1}, \ldots, P_{x-1} \in B_{W}$ wit $P_{i} \neq P_{j}$ for all $i \neq j$. Set $G^{\prime}:=\left\{g \in G: g\left(P_{i}\right)=P_{i}\right.$ for $1 \leq i \leq x-1\}$. Set $A:=H^{0}\left(\mathbf{P}^{n}, \mathcal{I}_{\left\{P_{1}, \ldots, P_{s-1}\right\}}(d)\right)$. $A$ induces a permutation group $G^{\prime \prime}$ on $B_{W} \backslash\left\{P_{1}, \ldots, P_{x-1}\right\}$. Since $G$ is $\left.x-1\right)$-transitive to show that $G$ is $x$-transitive it is sufficient to show that $G^{\prime \prime}$ is transitive. Since $W$ is general and $x \leq\left(\begin{array}{c}n+d \\ n\end{array}\right)$, we may see $P_{1}, \ldots, P_{s+1}$ as $x+1$ general points of $\mathbf{P}^{n}$. Set $D:=\left\langle\left\{P_{s}, P_{s+1}\right\}\right\rangle$. Hence $W \in A$, $\operatorname{dim}(A)=\left(\begin{array}{c}n+d \\ n\end{array}\right)-s+1$ and there is an irreducible family $\left\{V_{t}\right\}_{t \in T}, T$ a non-empty open subset of the line $D, V_{t} \in A$ for all $t \in T$, such that $V_{P_{s}}=W, t \in B_{V_{t}}$ for all $t \in T$, and $h^{1}\left(\mathbf{P}^{n}, \mathcal{I}_{\left\{P_{1}, \ldots, P_{s}, t\right\}}(d)\right)=0$ for all $t \in T$. This family shows that $G^{\prime \prime}$ is transitive, proving the Claim.

Recall that $W$ is general in $\Gamma_{n, d}$. By the Claim it is sufficient to show the existence of $S \subset B_{W}$ such that $\sharp(S)=s$. Then $h^{0}\left(\mathbf{P}^{n}, \mathcal{I}_{S}(d-1)\right)=0$, $h^{1}\left(\mathbf{P}^{n}, \mathcal{I}_{S}(t)\right)=0$ for all $t \geq d, h^{0}\left(\mathbf{P}^{n}, \mathcal{I}_{S}(d)\right)=\left(\begin{array}{c}n+d \\ n\end{array}\right)-s$ and $S$ is the schemetheoretic base locus of the linear system $\left|\mathcal{I}_{S}(d)\right|$. Since $h^{0}\left(\mathbf{P}^{n}, \mathcal{I}_{B_{W}}(d)\right)=n$, it is obvious the existence of $S \subset B_{W}$ such that $\sharp(S)=s$. Then $h^{0}\left(\mathbf{P}^{n}, \mathcal{I}_{S}(d-1)\right)=$ $0, h^{1}\left(\mathbf{P}^{n}, \mathcal{I}_{S}(t)\right)=0$ for all $t \geq d, h^{0}\left(\mathbf{P}^{n}, \mathcal{I}_{S}(d)\right)=\left(\begin{array}{c}n+d \\ n\end{array}\right)-s$. For any such $S$ the scheme-theoretic base locus $\Delta$ of the linear system $\left|\mathcal{I}_{S}(d)\right|$ is strictly contained in $B_{W}$. A weak form of the Cayley-Bacharach property gives $\sharp\left(B_{W} \backslash \Delta\right) \geq 2$. Fix $P \in B_{W} \backslash S$. Since $G$ is $\left(d^{n}-2\right)$-transitive and $\sharp\left(B_{W} \backslash \Delta\right)$, there is $g \in G$ such that $g(S)=S$ and $g(P) \in \Delta$. Hence $P \in \Delta$, contradiction.

Proof of Proposition 2. We use the set-up of the Proof of Proposition 1 with trivial modifications. For instance, we intersect every base locus with $Z_{1}$ and instead of $\Gamma_{n, d}$ we take an open subset of the Grassmannian of all $(n-$ $\left.\left(\begin{array}{c}n+s \\ n\end{array}\right)+s\right)$-dimensional linear subspaces of $H^{0}\left(\mathbf{P}^{n}, \mathcal{O}_{\mathbf{P}^{n}}(d+e)\right)$. The assumption $\gamma \geq 25$ allows us to use the Claim of the proof of Proposition 1, without doing then by hands the few exceptional cases.

Proof of Theorem 1. The statement of Theorem 1 is just an axiomatization of the proof of Proposition 1. 


\section{REFERENCES}

[1] J. Rathmann, The uniform position principle for curves in characteristic $p$, Math. Ann. 276 (1987), no. $4,565-579$.

[2] Z. C. Teitler, On the intersection of curves through a set of points in $\mathbb{P}^{2}$, J. Pure Appl. Algebra 209 (2007), no. 2, 571-581.

Received: May 23, 2007 